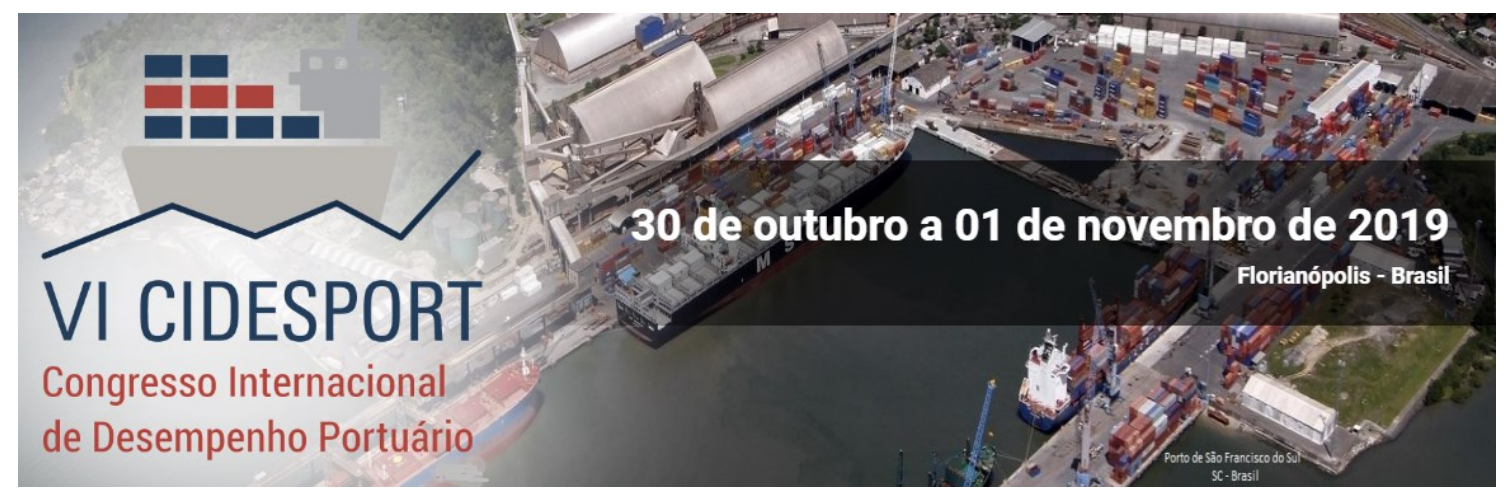

\title{
O EFEITO DA DISTÂNCIA NO FLUXO DE CARGAS - UMA ANÁLISE DA ESTRUTURA ESPACIAL DA HINTERLÂNDIA DOS PORTOS BRASILEIROS
}

\author{
Pedro Henrique Monteiro da Silva \\ Universidade Federal da Paraíba
}

Maria Silene Alexandre Leite

Universidade Federal da Paraílba

Raphael Kramer

Universidade Federal da Paraíba

Resumo: O Brasil passou por um processo de expansão econômica durante as primeiras décadas do século XXI, elevando seu PIB em $378 \%$ e saltando da $11^{\circ}$ para $5^{\circ}$ posição no ranking das economias globais. Tal crescimento econômico foi acompanhado pela expansão da movimentos de bens pelos portos brasileiros. No país, 95\% de toda carga movimentada nos portos nacionais passam por 19 complexos portuários, menos da metade dos 40 distribuídos ao longo da costa e do interior do Brasil. Estes portos competem entre si pelo tráfego de cargas do interior do território nacional e para o interior do território nos casos exportação e importação respectivamente. Com o objetivo de se estudar a distribuição de cargas dos portos para o interior do país este trabalho se propõe a analisar a estrutura espacial da hinterland dos portos brasileiros. Para tanto foram selecionados quatro grandes grupos de carga: Granel sólido agrícola, granel sólido não agrícola, granel líquido e carga geral e por meio de modelos de interação espacial foi estudado sua distribuição para as 137 mesorregiões do país. As cargas de granel agrícola e não agrícola aproximadamente $62 \%$ dos portos entregam mais que $60 \%$ da carga para o estado aonde está localizado o porto e aqueles que fazem fronteira com os mesmos. Os itens carga geral e granel líquido a apenas $15 \%$ dos portos entregam mais que $60 \%$ da carga para o estado aonde está localizado o porto e aqueles que fazem fronteira com os mesmos.

\section{INTRODUÇÃO}

Desde o início do século XXI o Brasil passou por um processo de expansão econômica. Segundo os dados oficiais do Instituto Brasileiro de Geografia e Estatística (IBGE), o PIB nacional passou por um aumento $378 \%$ em quinze anos. Partindo de aproximadamente 523 bilhões de dólares, para 1,98 trilhões de dólares no ano de 2016.

Esses números sozinhos poderiam não significar muito, se não houvesse também uma melhora relativa a outros países no mundo, porém, de acordo o banco de dados do Fundo Monetário Internacional (FMI) no mesmo período o país saltou da $11^{\circ}$ economia do mundo para a $5^{\circ}$ colocação, deixando para trás países como França, Rússia e Reino Unido.

${ }^{*}$ A revisão gramatical, ortográfica, ABNT ou APA foi realizada pelos autores. 
Tal crescimento econômico foi acompanhado pela expansão da movimentos de bens pelos portos brasileiros. Os números referentes as movimentações antes do ano de 2010 não estão disponíveis na Agência Brasileiras de Transportes Aquaviários (ANTAQ), que é o órgão governamental responsável pela fiscalização das atividades do setor. Porém pode-se observar que no período de 2010 à 2017 os portos brasileiros viram um aumento consistente de movimentação de cargas da ordem de $5 \%$ anualmente A figura 1 mostra a evolução da movimentação de cargas dos cinco maiores portos brasileiros no período de 2010 à 2017.

Figura 1- Movimentação em toneladas dos cinco maiores complexos portuários brasileiros nos últimos sete anos (fonte Estatísticos Aquaviário da ANTAQ)

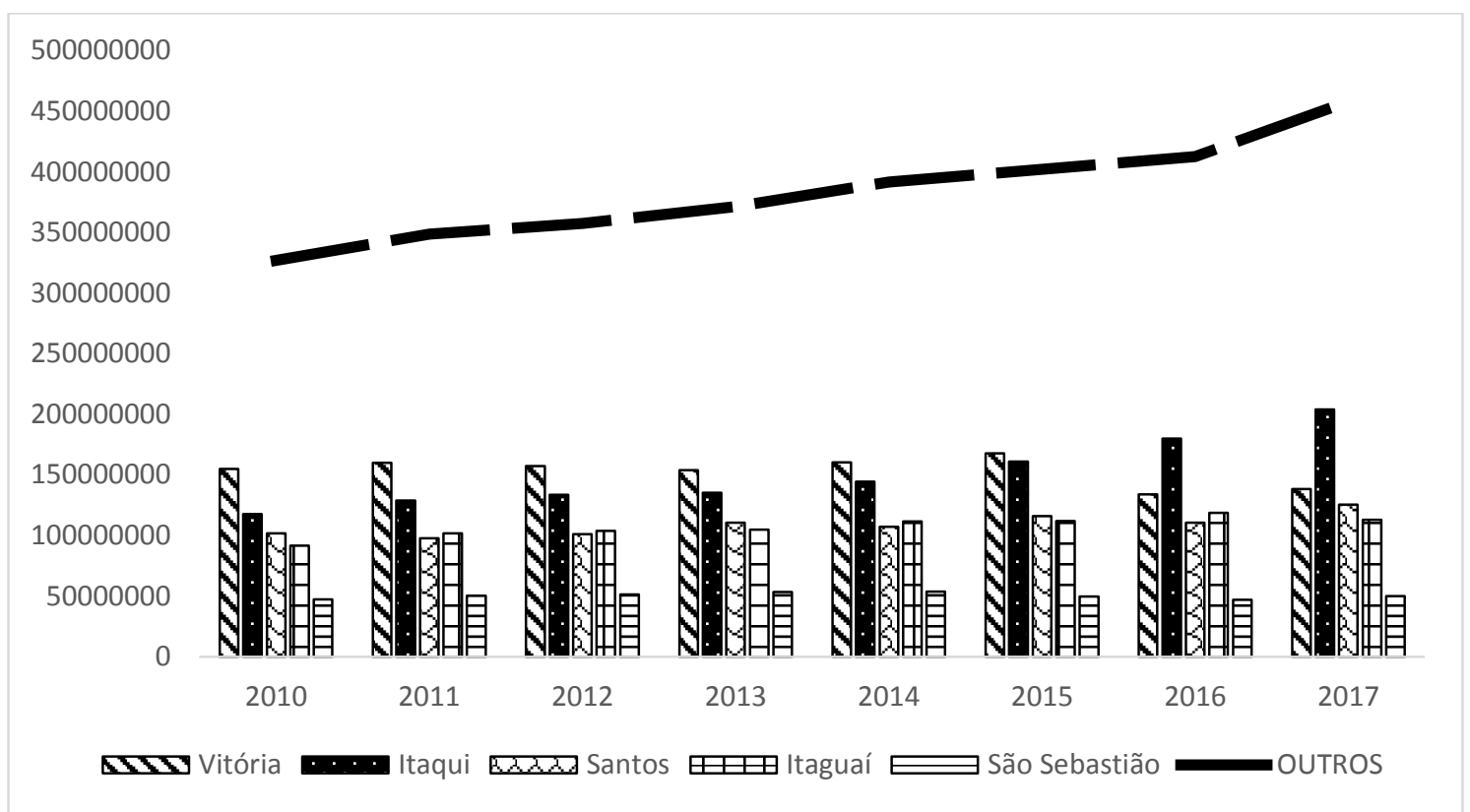

Fonte: Elaborado pelo autor

Neste período os cinco maiores portos nacionais foram responsáveis pela movimentação de $60 \%$ de toda a carga que entrou ou deixou o país, outro fato a ser destacado é que $95 \%$ de toda carga movimentada nos portos nacionais passam por 19 complexos portuários, menos da metade dos 40 distribuídos ao longo da costa e do interior do Brasil. Estes portos competem entre si pelo tráfego de cargas do interior do território nacional e para o interior do território nos casos exportação e importação respectivamente.

O interior do território também pode ser chamado de hinterland. Definimos hinterland como a zona geográfica circundando o complexo portuário de onde o mesmo coleta ou distribui as cargas que opera (GUERRERO, 2014). Inicialmente pode-se pensar que a hinterland refere-se apenas porção de terra mais próxima do porto, porém na realidade a hinterland de um porto pode ser tão ampla quanto possível e existir interseção entre as hinterlands de diferentes portos. Este fenômeno discutido anteriormente por Guerrero (2014) e Levine et al (2009).

O desenvolvimento portuário e sua integração com a hinterland são foco dos estudos de Garcia-Alonso, Martinez-Pardo e Vallejo-Pinto (2016); Lonza e Marolda (2016); Sugawara (2017). Para esses autores assim como para Ojadi e Walters (2015) os custos logísticos são um dos principais fatores afetando o desempenho portuário. Esses custos são diretamente proporcionais a distância dos portos aos destinos e origens das mercadorias que eles movimentam, trabalhos como os de Talley e $\mathrm{Ng}$ 


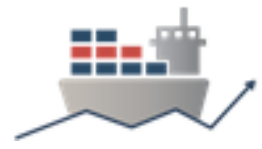

(2013) mostram que a proximidade é um dos principais critérios envolvidos no processo de escolha do porto. Logo se espera que quanto maior a distância de uma região até um dado porto, menor o fluxo de carga entre as duas localidades. Esse pensamento é corroborado entre outros trabalhos pelos os achados de Levine et al., (2009) que mostra como o fluxo de cargas decai em função da distância nos Estados Unidos. O mesmo foi demonstrado por Guerrero (2014) na França e Wang, Goodchild e Wang (2017) na China.

\section{REVISÃO DA LITERATURA}

Em se tratando do escopo da hinterland dos portos, um abundante número de trabalhos foi encontrado. Estudos abordando o assunto por diferentes perspectivas Jiaoe, Jingjuan e Li (2018) propuseram um modelo organizacional baseado na distância entre os nós e o custo de transporte. Talley e Ng (2017) trataram do assunto pela ótica dos determinantes da escolha das cadeias de suprimento, ou Yang, Luo e Ji (2016) que usou um modelo espacial temporal aplicado no caso do porto de Shangai, China. Wang, Meng e Miao (2016) fazendo uso de um modelo geométrico baseado no fluxo de cargas intermodal integrado com a questão da escolha das cadeias de suprimento, aplicando o modelo em dois portos chineses. Porém, a delimitação desse escopo por meio de uma abordagem espacial é escassa na literatura.

Durante o desenvolvimento deste trabalho apenas três artigos foram encontrados que tratavam diretamente a problemática da distribuição de cargas na hinterland pela ótica do modelo de interação espacial. São eles: Ferrari, Parola, e Gattorna (2011); Grecco, Moura, Garcia-alonso, e Salas-olmedo (2017) e Guerrero (2014).

Ferrari et al (2011), usou um modelo derivado do modelo gravitacional duplamente restringido, distância euclidiana e dados referentes a cargas conteinerizadas para delimitar a hinterland dos portos de Liguria, uma região no norte da Itália. Esses portos estão envolvidos em um contexto de alta competitividade não só com outros portos italianos, mas também portos maiores localizados no norte da Europa.

Similar ao trabalho anterior, Guerrero (2014) também usou um modelo derivado do modelo gravitacional duplamente restringido e carga conteinerizada, porém ao invés de distância euclidiana o autor usou o tempo de viagem de caminhão entre os nós. Foram examinados os dezesseis maiores portos franceses e cinco portos estrangeiros, que combinados movimentam $98 \%$ das cargas importadas na França e $97 \%$ das cargas exportadas. Esse trabalho faz uma análise tanto da hinterland quanto da foreland - equivalente da primeira, porém no exterior.

Grecco (2017), usou restrições duplas, tempo de viagem de caminhão, similar a Guerrero (2014), e propôs uma alteração ao modelo Huff - comumente utilizado para estimar a demanda em estabelecimentos comerciais - para ser usado como base para delimitação das hinterlands dos portos. Como validação do método ele aplicou esse modelo alterado no caso espanhol no contexto de cargas conteineirizadas.

Trabalhos focados em delimitar a hinterland dos portos brasileiros em escala nacional não foram encontrados durante a realização da revisão da literatura, os trabalhos encontrados como os de Azenha, Renato e Pequeno (2016) e Scavarda e Paiva (2010) delimitaram a hinterland de um único porto. Assim diferentemente das pesquisas ora apresentadas, este trabalho se propõe a analisar a estrutura espacial 
da hinterland dos portos brasileiros em escala nacional e investigar se o fenômeno do decaimento de fluxo também acontece no Brasil.

Para tanto foram selecionados quatro grandes grupos de carga: Granel sólido agrícola, granel sólido não agrícola, granel líquido e carga geral e por meio de modelos de interação espacial foi estudado sua distribuição para as 137 mesorregiões do país. A determinação desse fluxo de carga pode ser um ponto de partida para a tomada de decisão no que diz respeito a investimentos de infraestrutura e desenvolvimento portuário de forma consistente com as reais necessidades do panorama nacional. Ademais a importância do tema é elevada pelo contexto no qual estão envolvidos os portos brasileiros embora seja uma das maiores economias do mundo a infraestrutura de escoamento do país é sucateada, como explorado nos trabalhos de Filho (2001) e Scavarda e Paiva (2010).

\section{METODOLOGIA}

Dois grandes órgãos do governo federal serviram como origem para os dados brutos analisados nesta pesquisa. Os dados de movimentação portuária disponíveis no site oficial da Agência Nacional de Transportes Aquaviários - ANTAQ e a matriz origem/destino de movimentação de cargas em termos de massa inter-regionais no país no ano de 2015, disponível no site da Empresa Brasileira de Logística - EPL.

A matriz origem/destino (O/D) é categorizada pela EPL pela movimentação entre cidades. Para tornar o trabalho mais objetivo estas cidades foram agrupadas nas suas respectivas 167 mesorregiões, como encontradas nos mapas oficiais do Instituto Brasileiro de Geografia e Estatística - IBGE. Esta classificação resulta em uma matriz O/D de 167 por 167. A distribuição interporto foi ignorada, já que o objetivo é delimitar a hinterland de cada porto, similar ao feito no trabalho de Guerrero (2014). Uma versão simplificada da matriz O/D é apresentado na Figura 2.

Figura 2 - Representação da matriz O/D

\begin{tabular}{|c|c|c|c|c|c|}
\hline \multirow[t]{2}{*}{$\mathrm{De}$} & \multicolumn{5}{|l|}{ Para } \\
\hline & Porto 1 & Porto 2 & Porto ... & Porto j & Meso 1 \\
\hline Porto 1 & 0 & 0 & 0 & 0 & $V_{11}$ \\
\hline Porto 2 & 0 & 0 & 0 & 0 & $V_{21}$ \\
\hline Porto... & 0 & 0 & 0 & 0 & $\ldots$ \\
\hline Porto i & 0 & 0 & 0 & 0 & $V_{i 1}$ \\
\hline Meso 1 & $V_{11}$ & $V_{12}$ & $\ldots$ & $V_{1 j}$ & 0 \\
\hline Meso 2 & $V_{21}$ & $V_{22}$ & $\ldots$ & $V_{2 j}$ & 0 \\
\hline Meso ... & $\ldots$ & $\ldots$ & $\ldots$ & $\ldots$ & 0 \\
\hline Meso i & $V_{i 1}$ & $V_{i 2}$ & $\ldots$ & $V_{i j}$ & 0 \\
\hline Total & T porto 1 & T porto 2 & $\ldots$ & $\mathrm{T}$ porto $\mathrm{n}$ & T meso 1 \\
\hline
\end{tabular}

Fonte: Elaborado pelo autor

São quatro tipos de carga disponíveis na matriz O/D: Carga granel sólida agrícola - GSA; carga granel sólida não agrícola - GSNA; carga geral - CG e carga granel liquida - GL. Cada uma das cargas é composta como se segue: GSA - farelo de soja, milho e soja em grão. GSNA - carvão mineral, cimento, minério de ferro e 
outros minerais. CG - alimentos e bebidas (processados), celulose e papel, produtos básicos de borracha, plástico e não metálicos, produtos de exploração florestal silvicultura e manufaturados. GL - Combustíveis, petro e químicos.

Com o objetivo de escolher os principais portos nacionais para realização do estudo, os dados de movimentação portuária entre os anos de 2010 e 2016 foram coletados no site da ANTAQ. Então foram selecionados 18 terminais portuários distribuídos pelas quatro macrorregiões do Brasil, que de acordo com esses dados são responsáveis pela movimentação de $95 \%$ de toda carga que entra ou sai do país. Sete terminais estão localizados na região sudeste, cinco na região sul, cinco na região nordeste e quatro na região norte, sendo dois destes últimos portos fluviais.

Figura 2(a) - Localização geográfica dos portos selecionados para o estudo - Figura2(b) Representação gráfica do tamanho dos portos selecionados, baseado no volume de carga movimentado nos últimos sete anos
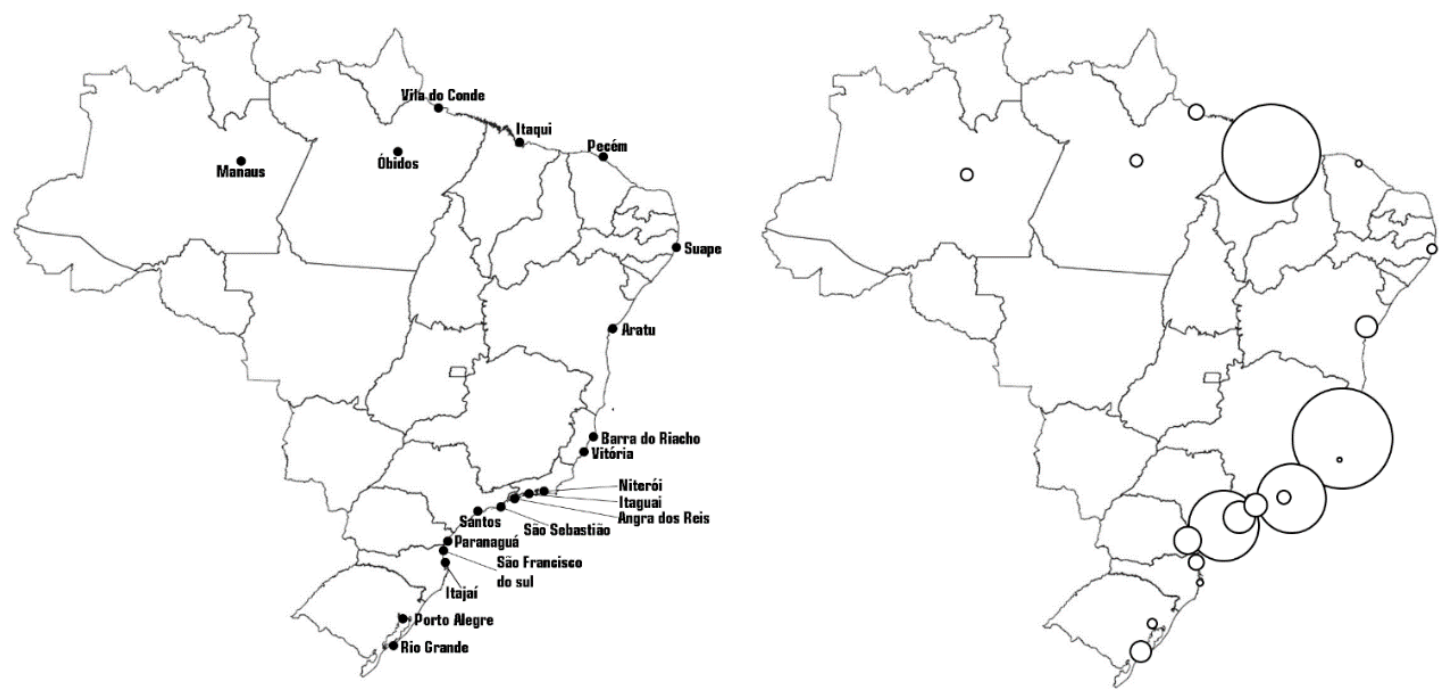

Fonte: Elaborado pelo autor

O fluxo de carga do porto de origem $\mathrm{i}$ até a área de destino $\mathrm{j}$ é dado por $\mathrm{V}_{\mathrm{ij}}$, a carga total que sai de um porto i para qualquer região j é dado por $\mathrm{O}_{\mathrm{i}}$, e a carga total que chega uma determinada região $\mathrm{j}$ tendo origem de qualquer porto i é dado por $D_{j}$.

$$
\begin{aligned}
& O_{i}=\sum_{j} V_{i j} \\
& D_{j}=\sum_{i} V_{i j}
\end{aligned}
$$

Para se realizar a delimitação da hinterland dos portos nacionais é adotada a análise de concentração espacial, à luz do estudo feito por Wang, Goodchild, e Wang (2017). Esta análise é baseada em quatro parâmetros, o índice de HirschmanHerfindahl (HHI), aqui referido como HP, o quociente de localização (LQ), aqui tratado como LP e duas razões de importação/distribuição, $\mathrm{P}_{\mathrm{i}}{ }^{\mathrm{Ai}}$ e $\mathrm{R}_{\mathrm{i}}$ 


$$
\begin{gathered}
H P_{i}=\sum_{j}\left[\frac{V_{i j}}{\sum_{j} V_{i j}}\right]^{2} \\
L P_{i j}=\frac{V_{i j} / \sum_{i} V_{i j}}{\sum_{j} V_{i j} / \sum_{i} \sum_{j} V_{i j}}
\end{gathered}
$$

O índice $\mathrm{HP}$ i é diretamente proporcional ao nível de concentração da hinterland de um dado porto, esta variável em conjunto com o LP $\mathrm{ij}$, que mensura a concentração da distribuição de carga de um dado porto i para uma dada região j, quando $L P_{i j}>1$ significa que essa região pode ser definida como sendo primordial para a hinterland deste porto. Quanto maior o número de regiões com $L P_{i j}>1$, mais decentralizada a hinterland do porto (Wang, Goodchild, e Wang , 2017).

$$
\begin{aligned}
P_{i}^{A 1} & =\frac{\sum_{j \epsilon A 1} V_{i j}}{\sum_{j} V_{i j}} \\
P_{i}^{A 2} & =\frac{\sum_{j \epsilon A 2} V_{i j}}{\sum_{j} V_{i j}}
\end{aligned}
$$

$\mathrm{O}$ índice $\mathrm{Pi}_{\mathrm{i}}^{\mathrm{Ai}}$ é separado em duas razões, $\mathrm{Pi}^{\mathrm{A} 1}$ e $\mathrm{P}_{\mathrm{i}}^{\mathrm{A} 2}$, essas razões são definidas de tal forma que representam a proporção de carga distribuída a partir de um dado porto i para dois grupos regionais, A1 que é o estado o qual o porto pertence e A2 que são os estados adjacentes a este. Caso $\mathrm{Pi}^{\mathrm{A} 1}+\mathrm{P}^{\mathrm{A} 2}$ 0,99 temos uma hinterland concentrada, caso essa inequação seja falsa temos uma hinterland descentralizada (Wang, Goodchild, e Wang , 2017).

$$
R_{i}=\frac{\sum_{j \epsilon r 1} V_{i j}}{\sum_{j \epsilon r 1} D_{j}}
$$

Este índice denota a capacidade que cada porto tem para atrair as cargas da macrorregião onde estão localizadas (Wang, Goodchild, e Wang , 2017).

\section{RESULTADOS}

Uma análise de concentração espacial foi conduzida nos dezoito maiores portos do país para identificar o fenômeno da distância-decaimento no fluxo de cargas nos portos nacionais. As tabelas 1, 2, 3 e 4 mostram os portos divididos por região e apresentam os resultados para análise de concentração espacial para os quatro tipos de carga - GSA, GSNA, CG e GL. 
VI CIDESPORT/2019

Congresso Internacional

de Desempenho Portuário

Tabela 1 - Parâmetros de concentração espacial para as granel sólido agrícola

\begin{tabular}{|c|c|c|c|c|c|}
\hline \multicolumn{6}{|c|}{ Granel Sólido Agrícola } \\
\hline Região & Porto & $\mathrm{P} 1$ & $\mathrm{P} 2$ & Hpi & $\mathrm{Ri}$ \\
\hline \multicolumn{6}{|c|}{ Nordeste } \\
\hline & Itaqui & 0,52 & 0,04 & 0,19 & 0,06 \\
\hline & Aratu - Salvador & 0,35 & 0,30 & 0,10 & 0,22 \\
\hline & Suape - Recife & 0,29 & 0,30 & 0,14 & 0,30 \\
\hline & Pecém - Fortaleza & 0,60 & 0,09 & 0,13 & 0,21 \\
\hline \multicolumn{6}{|l|}{ Norte } \\
\hline & Vila do Conde - Belém & 0,63 & 0,00 & 0,38 & 0,05 \\
\hline & Óbidos & 0,00 & 0,05 & 0,30 & 0,02 \\
\hline & Manaus & 0,03 & 0,97 & 0,99 & 0,07 \\
\hline \multicolumn{6}{|l|}{ Sudeste } \\
\hline & Vitória & 0,08 & 0,30 & 0,15 & 0,04 \\
\hline & Santos & 0,70 & 0,20 & 0,31 & 0,62 \\
\hline & Itaguaí - Niterói & 0,16 & 0,50 & 0,29 & 0,02 \\
\hline & São Sebastião & 0,08 & 0,70 & 0,27 & 0,01 \\
\hline & Angra dos Reis & 0,06 & 0,92 & 0,30 & 0,14 \\
\hline & Barra do Riacho & 0,40 & 0,19 & 0,45 & 0,00 \\
\hline \multicolumn{6}{|l|}{ Sul } \\
\hline & Paranaguá - Antonina & 0,20 & 0,35 & 0,60 & 0,21 \\
\hline & Rio Grande & 0,89 & 0,00 & 0,81 & 0,30 \\
\hline & São Francisco do Sul & 0,64 & 0,26 & 0,43 & 0,14 \\
\hline & Porto Alegre & 0,37 & 0,17 & 0,47 & 0,13 \\
\hline & Itajaí & 0,49 & 0,18 & 0,21 & 0,05 \\
\hline
\end{tabular}

Fonte: Elaborado pelo autor 
VI CIDESPORT/2019

Congresso Internacional

de Desempenho Portuário

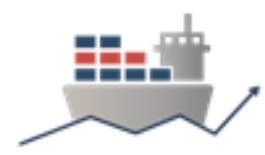

Tabela 2 - Parâmetros de concentração espacial para as granel sólido não agrícola

\begin{tabular}{|c|c|c|c|c|c|}
\hline \multicolumn{6}{|c|}{ Granel Sólido Não Agrícola } \\
\hline Região & Porto & $\mathrm{P} 1$ & P2 & Hpi & $\mathrm{Ri}$ \\
\hline \multicolumn{6}{|c|}{ Nordeste } \\
\hline & Itaqui & 0,44 & 0,23 & 0,83 & 0,04 \\
\hline & Aratu - Salvador & 0,19 & 0,27 & 0,04 & 0,04 \\
\hline & Suape - Recife & 0,39 & 0,47 & 0,13 & 0,08 \\
\hline & Pecém - Fortaleza & 0,20 & 0,36 & 0,05 & 0,05 \\
\hline \multicolumn{6}{|l|}{ Norte } \\
\hline & Vila do Conde - Belém & 0,95 & 0,00 & 1,00 & 0,01 \\
\hline & Óbidos & 0,00 & 0,55 & 0,59 & 0,94 \\
\hline & Manaus & 0,34 & 0,53 & 0,25 & 0,01 \\
\hline \multicolumn{6}{|l|}{ Sudeste } \\
\hline & Vitória & 0,05 & 0,07 & 0,96 & 0,11 \\
\hline & Santos & 0,56 & 0,15 & 0,19 & 0,23 \\
\hline & Itaguaí - Niterói & 0,50 & 0,30 & 0,95 & 0,09 \\
\hline & São Sebastião & 0,71 & 0,13 & 0,09 & 0,06 \\
\hline & Angra dos Reis & 0,22 & 0,53 & 0,09 & 0,10 \\
\hline & Barra do Riacho & 0,39 & 0,05 & 0,24 & 0,04 \\
\hline \multicolumn{6}{|l|}{ Sul } \\
\hline & Paranaguá - Antonina & 0,27 & 0,45 & 0,05 & 0,26 \\
\hline & Rio Grande & 0,12 & 0,16 & 0,45 & 0,26 \\
\hline & São Francisco do Sul & 0,64 & 0,12 & 0,18 & 0,08 \\
\hline & Porto Alegre & 0,67 & 0,12 & 0,10 & 0,15 \\
\hline & Itajaí & 0,56 & 0,08 & 0,10 & 0,11 \\
\hline
\end{tabular}

Fonte: Elaborado pelo autor 
VI CIDESPORT/2019

Congresso Internacional

de Desempenho Portuário

Tabela 3 - Parâmetros de concentração espacial para a carga geral

\begin{tabular}{|c|c|c|c|c|c|}
\hline \multicolumn{6}{|c|}{ Carga Geral } \\
\hline Região & Porto & P1 & P2 & Hpi & $\mathrm{Ri}$ \\
\hline \multicolumn{6}{|c|}{ Nordeste } \\
\hline & Itaqui & 0,03 & 0,08 & 0,03 & 0,02 \\
\hline & Aratu - Salvador & 0,08 & 0,24 & 0,03 & 0,10 \\
\hline & Suape - Recife & 0,08 & 0,19 & 0,03 & 0,08 \\
\hline & Pecém - Fortaleza & 0,03 & 0,11 & 0,03 & 0,07 \\
\hline \multicolumn{6}{|l|}{ Norte } \\
\hline & Vila do Conde - Belém & 0,11 & 0,11 & 0,08 & 0,01 \\
\hline & Óbidos & 0,05 & 0,06 & 0,12 & 0,01 \\
\hline & Manaus & 0,01 & 0,06 & 0,14 & 0,05 \\
\hline \multicolumn{6}{|l|}{ Sudeste } \\
\hline & Vitória & 0,02 & 0,15 & 0,05 & 0,05 \\
\hline & Santos & 0,39 & 0,26 & 0,05 & 0,37 \\
\hline & Itaguaí - Niterói & 0,08 & 0,46 & 0,08 & 0,06 \\
\hline & São Sebastião & 0,38 & 0,27 & 0,02 & 0,04 \\
\hline & Angra dos Reis & 0,09 & 0,47 & 0,02 & 0,07 \\
\hline & Barra do Riacho & 0,07 & 0,07 & 0,03 & 0,02 \\
\hline \multicolumn{6}{|l|}{ Sul } \\
\hline & Paranaguá - Antonina & 0,09 & 0,44 & 0,04 & 0,10 \\
\hline & Rio Grande & 0,16 & 0,09 & 0,19 & 0,03 \\
\hline & São Francisco do Sul & 0,10 & 0,14 & 0,05 & 0,07 \\
\hline & Porto Alegre & 0,14 & 0,10 & 0,09 & 0,11 \\
\hline & Itajaí & 0,13 & 0,11 & 0,09 & 0,06 \\
\hline
\end{tabular}

Fonte: Elaborado pelo autor 
VI CIDESPORT/2019

Congresso Internacional

de Desempenho Portuário

Tabela 4 - Parâmetros de concentração espacial para as granel líquido

\begin{tabular}{|c|c|c|c|c|c|}
\hline \multicolumn{6}{|c|}{ Granel Líquido } \\
\hline Região & Porto & P1 & P2 & Hpi & $\mathrm{Ri}$ \\
\hline \multicolumn{6}{|c|}{ Nordeste } \\
\hline & Itaqui & 0,25 & 0,41 & 0,07 & 0,04 \\
\hline & Aratu - Salvador & 0,20 & 0,23 & 0,13 & 0,04 \\
\hline & Suape - Recife & 0,23 & 0,42 & 0,09 & 0,08 \\
\hline & Pecém - Fortaleza & 0,47 & 0,09 & 0,10 & 0,05 \\
\hline \multicolumn{6}{|l|}{ Norte } \\
\hline & Vila do Conde - Belém & 0,21 & 0,40 & 0,15 & 0,01 \\
\hline & Óbidos & 0,03 & 0,78 & 0,62 & 0,94 \\
\hline & Manaus & 0,04 & 0,32 & 0,14 & 0,01 \\
\hline \multicolumn{6}{|l|}{ Sudeste } \\
\hline & Vitória & 0,43 & 0,06 & 0,09 & 0,06 \\
\hline & Santos & 0,41 & 0,24 & 0,07 & 0,24 \\
\hline & Itaguaí - Niterói & 0,19 & 0,37 & 0,29 & 0,37 \\
\hline & São Sebastião & 0,76 & 0,10 & 0,06 & 0,10 \\
\hline & Angra dos Reis & 0,09 & 0,47 & 0,36 & 0,47 \\
\hline & Barra do Riacho & 0,05 & 0,06 & 0,10 & 0,06 \\
\hline \multicolumn{6}{|l|}{ Sul } \\
\hline & Paranaguá - Antonina & 0,34 & 0,38 & 0,07 & 0,26 \\
\hline & Rio Grande & 0,37 & 0,05 & 0,10 & 0,26 \\
\hline & São Francisco do Sul & 0,41 & 0,14 & 0,08 & 0,08 \\
\hline & Porto Alegre & 0,36 & 0,08 & 0,08 & 0,15 \\
\hline & Itajaí & 0,65 & 0,05 & 0,34 & 0,11 \\
\hline
\end{tabular}

Fonte: Elaborado pelo autor

O fenômeno distância-decaimento afeta de forma diferente cada um dos tipos de carga estudados. As cargas de granel agrícola e não agrícola apresentam os maiores valores de índice HP, que é proporcional ao nível de concentração do porto. Nestes itens aproximadamente $62 \%$ dos portos entregam mais que $60 \%$ da carga para o estado aonde está localizado o porto e aqueles que fazem fronteira com os mesmos.

Os itens carga geral e granel líquido apresentam os menores índices HP, isso indica maior descentralização do porto, logo um menor impacto da distância no transporte de carga. Para estes tipos de carga apenas $15 \%$ dos portos entregam mais que $60 \%$ da carga para o estado aonde está localizado o porto e aqueles que fazem fronteira com os mesmos.

\section{CONCLUSÕES E SUGESTÕES PARA TRABALHOS FUTUROS}

Este trabalho através dos quatro índices - HP, $\mathrm{P}_{\mathrm{i}} \mathrm{A}^{\mathrm{A}}, \mathrm{P}_{\mathrm{i}} \mathrm{A}^{2}$, $\mathrm{LP}$ e $\mathrm{R}_{\mathrm{i}}$ - demonstrou como o fenômeno da distância-decaimento afeta quatros grandes grupos de carga nos principais portos nacionais. Também foi possível observar que cada uma delas é afetada pela distância em intensidades distintas - esses achados estão em par com outros trabalhos como os de Guerrero (2014), Levine (2009) e Wang (2017).

O resultado encontrado para granel agrícola e não agrícola são consistentes com os achados por Guerrero (2014), em sua investigação da hinterland francesa o autor 


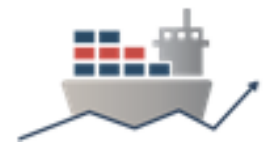

mostrou que a distância afeta fortemente o transporte desse tipo de produto. A forma como a distância afeta os produtos agrícolas é justificado pelo fato de os mesmos serem commodities com baixo valor agregado que não suportam agregar custos logísticos elevados associados ao transporte em longas distância. Essa afirmação é ainda mais relevante frente a realidade das estradas nacionais. Este tipo de carga é trabalhado principalmente pelos portos de Santos, Amazonas e Paranaguá, os três com parcelas similares da demanda. A posição central dos centros produtos de produtos agrícolas no país pode ser uma das explicações para tal fenômeno, assim como o recente movimento para o norte das culturas de soja.

A carga geral, que é composta por alimentos e bebidas (processados), celulose e papel, produtos básicos de borracha, plástico e não metálicos, produtos de exploração florestal silvicultura e manufaturados, apresentou os menores valores de HP para os portos estudados, indicando forte descentralização no tratamento de sua carga. A distribuição desta é bem dividida pelos portos estudados e é pouco afetada pela distância. Resultado este também em par com o encontrado por Guerrero (2014).

A única carga que apresentou comportamento, frente ao efeito da distância, distinto do estudado por Guerrero (2014) foi a carga de granel líquido - combustíveis, petro e químicos. No estudo realizado pelo autor francês, esta carga sofria a maior influência da distância, ela apresentava o decaimento mais rápido que todas as outras estudadas. No caso do Brasil, esta carga possui valores de HP próximos aos encontrados na carga geral, logo é uma carga com característica de descentralização nos portos do país

Embora significantes esses resultados não são suficientes para se fazer uma delimitação completa da hinterland nacional, a pesquisa precisa ser continuada com o objetivo de se usar o modelo gravitacional para se estudar o fenômeno do decaimento da distância em sua totalidade e verificar se os resultados encontrados aí estarão em par com os achados em outros trabalhos. De posse desse modelo é possível extrapolar os resultados e delimitar mais precisamente a hinterland dos outros portos nacionais, assim como prover uma forma de se estimar os fluxos de carga entre os portos e as mesorregiões nacionais.

\section{REFRERÊNCIAS}

AZENHA, C.; RENATO, L.; PEQUENO, B. O PORTO DO PECÉM - CE E AS SUAS RELAÇÕES COM O BRASIL : DEFINIÇÃO DE UMA HINTERLÂNDIA. p. 44-62, 2016.

FERRARI, C.; PAROLA, F.; GATTORNA, E. Measuring the quality of port hinterland accessibility: The Ligurian case. Transport Policy, v. 18, n. 2, p. 382-391, 2011.

FILHO, A. G. Melhoramentos, reaparelhamentos e modernização dos portos brasileiros : a longa e constante espera 1. v. 1910, n. 1, p. 455-489, 2007.

FMI. World economic outlook database. Disponível em: <www.imf.org>. Acesso em 13 de setembro de 2018.

GARCIA-ALONSO, L.; MARTINEZ-PARDO, A.; VALLEJO-PINTO, J. A. Analysis of the spatial development of the hinterland of ports: a case study. International Journal of Shipping and Transport Logistics, v. 8, n. 2, p. 111-128, 2016.

GRECCO, T. et al. Delimiting the scope of the hinterland of ports : Proposal and case study. Journal of Transport Geography, v. 65, n. September, p. 35-43, 2017. 
VI CIDESPORT/2019

Congresso Internacional

de Desempenho Portuário

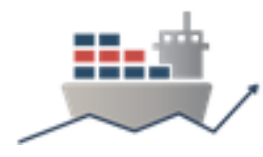

GUERRERO, D. Deep-sea hinterlands : Some empirical evidence of the spatial impact of containerization. v. 35, p. 84-94, 2014.

IBGE. Série histórica do PIB IBGE, 2017. Disponível em: <www.inge.gov.br>. Acesso em 13 de setembro de 2018.

JIAOE, W.; JINGJUAN, J.; LI, M. An organizational model and border port hinterlands for the China-Europe Railway Express. JOURNAL OF GEOGRAPHICAL SCIENCES, v. 28, n. 9 , p. 1275-1287, 2018.

LEVINE, B.; NOZICK, L.; JONES, D. Estimating an origin - destination table for US imports of waterborne containerized freight. Transportation Research Part E, v. 45, n. 4, p. 611-626, 2009.

LONZA, L.; MAROLDA, M. C. Ports as drivers of urban and regional growth. In: RAFALSKI, L.; ZOFKA, A. (Eds.). . Transport Research Arena Tra2016. [s.I: s.n.]. v. 14p. 2507-2516.

OJADI, F.; WALTERS, J. Critical factors that impact on the efficiency of the Lagos seaports. Journal of Transport and Supply Chain Management, v. 9, n. 1, p. 1-13, 2015.

SCAVARDA, L. F.; PAIVA, R. Zonas de influência portuárias - hinterlands: conceituação e metodologias para sua delimitação. p. 553-566, 2010.

SUGAWARA, J. Port and hinterland network: A case study of the Crescent Corridor intermodal freight program in the US. Transportation Research Procedia, v. 25, p. 916-927, 2017.

TALLEY, W. K.; NG, M. Int . J . Production Economics Maritime transport chain choice by carriers , ports and shippers. Intern. Journal of Production Economics, v. 142, n. 2, p. 311-316, 2013.

TALLEY, W. K.; NG, M. Hinterland transport chains: Determinant effects on chain choice. International Journal of Production Economics, v. 185, p. 175-179, 2017.

WANG, L.; GOODCHILD, A.; WANG, Y. The effect of distance on cargo flows : a case study of Chinese imports and their hinterland destinations. Maritime Economics \& Logistics, 2017.

WANG, X.; MENG, Q.; MIAO, L. Delimiting port hinterlands based on intermodal network flows: Model and algorithm. TRANSPORTATION RESEARCH PART ELOGISTICS AND TRANSPORTATION REVIEW, v. 88, p. 32-51, 2016.

YANG, J.; LUO, M.; JI, A. Analyzing the spatial-temporal evolution of a gateway's hinterland: A case study of Shanghai, China. TRANSPORTATION RESEARCH PART E-LOGISTICS AND TRANSPORTATION REVIEW, v. 95, p. 355-367, nov. 2016. 